\title{
ESTUDIOS
}

\section{Borges y Nouvelle Critique}

J. B. J'ai l'impression qu'on m'a lu en France, d'une façon tellement intelligente. Peut-être $m$ 'a ton lu avec plus d'intelligence que je n'ai mis à écrite, noi! J'ai l'impression qu'on m'a entichi un peu ou beaucoup en me lisant.

G. C. Nous avons l'impression de nous être enrichis en vous lisant!

J. B. Eh bien c'est réciproque, tant mieux! Mais quand $\mathrm{je}$ vois lẹs analyses qu'on a faites de mes nouvelles, comment on les a lues, comment on les a prises au sérieux, et comment, en même temps, on a senti ce qu'il y a d'humout, d'humour un peu secret peut-être...

Georges Charbonnier: Entrotiens avec Jorge Luis Borges (Paris, 1967)

Los franceses han sido los primeros viajeros no hispánicos en intentar una cartografía de esa terra incognita que cubre el nombre de Jorge Luis Borges. Ya en 1925, Valéry Larbaud, lector poligloto e impune de la nueva literatura, dedicaba un artículo al primer libro de ensayos, Inquisiciones, del entonces joven poeta argentino. Por esos mismos años, un joven de origen vasco-francés, Néstor Ibarra esbozaba una tesis universitaria para la Facultad de Filosofía y Letras de Buenos Aires sobre la nueva poesía argentina. En 1933, Drieu la Rochelle, al regresar de una visita a la Argentina en momentos en que todos discutían el último libro de ensayos de Borges (Discusión, 1932), descubría que "Borges vaut le voyage". En 1939, Ibarra publica en Mesures, 1a (tal vez) primera traducción de Borges en Francia: "El acercamiento a Almotásim". En plena guerra, Roger Caillois, que entonces reside en Buenos Aires, funda allí, con el patrocinio de Victoria Ocampo, una revista de la Francia Libre, Lettres françaises, donde se publicarán más traducciones de Borges y textos sobre su obra. ${ }^{1}$

1 Doy la bibliografía básica de estos primeros contactos franceses con la obra de Borges: . 
Después de la guerra comienza la avalancha: hay traducciones pot todas partes, y en particular en Cabiers du Sud, en La Nouvelle NRF, en Les Temps Modernes. En esta última revista, la más discutida en los años de la segunda posguerra, se publica un enciclopédico y provocativo artículo de Etiemble: "Un homme à tuer: J. L. Borges" (Paris, setiembre 1962), en que se discute el supuesto "cosmopolitismo" del autor argentino. En 1951, Caillois empieza a publicar, más o menos ordenadamente, en su colección "La Croix du Sud", de Gallimard, la traducción de los libros más importantes de Borges: en el primer volumen, Fictions, uno de los traductores es el ubicuo Néstor Ibarra; el último hasta la fecha, Oeuvre poétique 1925-1965, es de Ibarra solamente. ${ }^{2}$ Entre 1951 y 1970, fecha de este último volumen, la fortuna de Borges en Francia, y en el resto del mundo occidental, ha alcanzado proporciones incalculables. Ya en 1961, al recibir ex-aequo con Samuel Beckett el Premio Internacional de Editores, otorgado en Formentor, Borges empieza su firme carrera internacional. En la concesión de ese premio, Borges contó no sólo con el apoyo de los editores de habla española, sino con el imprescindible de los franceses. Tres años después del premio, el volu. men colectivo que le dedica L'Herne (marzo 1964) reúne más de 60 escritores y críticos de distintas partes del mundo para quienes Borges efectivamente vale el viaje. A partir de entonces, Borges se convierte no sólo en punto de referencia obligado cuando se trata de un cierto tipo de literatura (su nombre aparece frecuentemente asociado a los de Kafka o Nabokov) sino en punto de partida para especulaciones críticas como las efectuadas por Genette y Ricardou, como estímulo para la invención narrativa (Robbe-Grillet), filosófica (Michel Foucault), cinematográfica (Godard). Por eso es literalmente imposible trazar en un

Néstor Ibarta, La nueva poesia argentina (1921-1929), Buenos Aires, 1930.

Pierre Drieu la Rochelle, "Discusión sobre Jorge Luis Borges: Borges waut lr: voyage", en Megáfono, núm. 11, Buenos Aires, agosto 1933.

Jorge 'Luis Borges, "L'approche du caché" ("El acercamiento a Almotásim"), en Mesures, Paris: 1939 (trad. Néstor Ibarra).

Jorge Luis Borges, "Assyriennes" ("Ia lotería en Babilonia", "La biblioteca de Babel"), en Lettres françaises, núm. 14, Buenos Aires, octubre $1^{\circ} 1944$ (trad. N. Ibarra).

Néstor Ibarra, "Jorge Luis Borges", en Lettres françaises, núm. 14, Buenos Aires, octubre 191944.

2 La colaboración de Caillois e Ibarra produce varios volúmenes de Borges en francés que son muy accesibles ya que se reeditan constantemente. Quisiera señalar al lector uno menos conocido pero esencial para reconstruir la primera imagen francesa de Borges. Me refiero al libro de Ibarra, Borges et Borges (Paris: L'Herne, 1969), que bajo la forma de un diálogo del crítico con un interlocutor anónimo, permite a Ibarra opinar, en forma libre y llena de humor e ironía, sobre su amigo y maestro. Una versión anterior, y más breve, del diálogo se había publicado en el número de L'Herne sobre Borges (Paris, 1964). 
breve artículo el laberíntico diseño de la presencia de Borges en la cultura francesa de hoy. Desde la cita de un texto suyo con que inaugura Foucault su libro, Les mots e les cboses (1966) hasta la cita, no identificada, con que el cerebro electrónico de Alpbaville (1965) hace suyas unas palabras de la conclusión de "Nueva refutación del Tiempo", ${ }^{3}$ la fortuna de Borges en Francia cubre todos los campos y va de la Sorbonne a las fortalezas de la cultura pop.

De todo ese entrecruzado y caótico sistema de referencias, un núcleo de especulación crítica se destaca notablemente. Es el núcleo que se forma en torno de ciertas ideas de Borges sobre la narración y sobre su propia práctica de narrador. Tal vez convenga examinar con algún detalle las piczas fundamentales que componen ese núcleo y que, en su mayor parte, son obra de lo que podemos llamar por comodidad la Nouvelle critique.

\section{EL INFINITO LITERARIO}

Corresponde a uno de los maestros de esa nueva crítica, al inagotable Maurice Blanchot, el mérito de haber descubierto uno de los aspectos centrales del juego literario de Borges. En un ensayo que está recogido en Le livre à venir (Paris, 1959), pero que es seguramente de 1953, Blanchot ataca el centro de la weltanscbaunng literaria de Borges: la noción de infinito. ${ }^{4}$ Blanchot afirma:

Je soupçonne Borges d'avoir reçu l'infini de la littérature. Ce n'est pas pour faire entendre qu'il n'en a qu'une calme connaissance tirée d'oeuvres littéraires, mais pour affirmer que l'expérience de la littérature est peut-être fondamentalement proche des paradoxes et des sophismes de ce que Hegel, pour l'écarter, appelait le mauvais infini. (116)

Pasa luego Blanchot a demostrar que cualquier espacio limitado puede convertirse en infinito si se vuelve de golpe para nosotros un espacio oscuro, si la ceguera (real o metafórica) nos invade.

Pour l'homme mesuré et de mesure, la chambre, le désert et le monde sont des lieux strictement determinés. Pour l'homme déser-

3 Sobre este punto véase mi Borgès par lui-même (Paris: Editions du Seuil, 1970), p. 75 .

4 Maurice Blanchot, Le livre à venir (Paris: Gallimard, 1959), pp. 116-119. 
tique et labyrinthique, vouẻ à l'erreur d'une démarche nécessairement un peu plus longue que sa vie, le même space sera vraiment infini, même s'il sait qu'il ne l'est pas et d'autant plus qu'il le saura. (116)

Al definir así a Borges como un ser laberíntico, encerrado en el espacio que la oscuridad y la ceguera vuelven infinito, Blanchot sienta las bases para lo que sigue. Un somero análisis del espacio infinito permite a Blanchot mostrar que es una prisión de la que es imposible salir: no hay línea recta, no se va jamás de un punto a otro, no se parte jamás de un lugar para ir a otro, no hay punto de partida ni se puede dar comienzo a la marcha. Antes de empezar, ya se está volviendo a empezar, se vuelve antes de partir. Tal es el secreto del infinito "malo", de que hablaba Hegel, y que corresponde a la "mala" eternidad.

Borges, homme essentiellement littéraire (ce qui veut dire qu'il est toujours prêt à comprendre selon le mode de comprehénsion qu'autorise la littérature), est aux prises avec la mauvaise eternité et la mauvaise infinité, les seules peut-être dont nous puissions faire l'épreuve, jusqu'à ce glorieux retournement qui s'appelle l'extase. (117)

A1 insistir sobre el carácter esencialmente literario de Borges (el hombre y no sólo la obra), Blanchot prepara el terreno para examinar uno de los conceptos básicos de su mundo imaginario: la identificación del libro y el mundo. A primera vista, apunta Blanchot, esta identificación serviría para tranquilizar a un espíritu libresco, y sobre todo a un aficionado a esos libros de ficción, organizados hábilmente como problemas completamente oscuros a los que se encuentra soluciones completamente claras: las novelas policiales. Pero en Borges no hay tranquilidad posible.

Mais si le monde est un livre, tout livre est le monde, et de cette innocente tautologie, il résulte des conséquences redoutables.

Ceci d'abord, qu'il n'y a plus de borne de référence. Le monde et le livre se renvoient éternellement et infinitement leurs images réflétées. Ce pouvoir indéfini de miroitment, cette multiplication scintillante et illimitée - qui est le labyrinthe de la lumière et qui du reste n'est pas rien - sera alors tout ce que nous trouverons, vertigineusement, au fond de notre désir de comprendre. 
Ceci encore, que si le livre est la possibilité du monde, nous devons en conclure qu'est aussi à l'oeuvre dans le monde non seulement le pouvoir de faire, mais ce grand pouvoir de feindre, de truquer et de tromper dont tout ouvrage de fiction est le produit d'autant plus évident que ce pouvoir y sera mieux dissimulé. (117-118)

Del infinito "malo" de Hegel al infinito literario de Borges, Blanchot se mueve con la mayor sutileza de análisis. Su insistencia en el carácter literario de este hombre y esta obra no implica ninguna desvalorización, ni siquiera en el terreno moral. Por el contrario, Blanchot habrá de subrayar precisamente el valor moral de esta actitud.

Fictions, Artifices risquent d'être les noms les plus honnêtes que la littérature puisse recevoir; et reprocher à Borges d'écrire des récits qui répondent très bien à ces titres, c'est lui reprocher cet excès de franchise sans lequel la mystification se prend lourdement au mot (Schopenhauer, Valéry, on le voit, sont les astres qui brillent dans ce ciel privé de ciel). (118)

Escritas hacia 1953, estas palabras de Blanchot no fueron lamentablemente oídas por una generación de críticos argentinos, e hispano. americanos, que se lanzaron entonces a acusar a Borges de juego, de bizantinismo, de mala fe, en el sentido sartriano del término. La admiración, algo servil, de estos jóvenes críticos de entonces por la literatura francesa (todos habían leído y repetido los argumentos del debate sobre la literatura "engagée"), no les había enseñado a distinguir dentro de ella lo que era pensamiento original (Bachelard, Bataille, Blanchot, el primer Sartre) y lo que era tan sólo reflejo de una situación política de alcance muy limitado. Peto ésta es otra historia. El análisis de Blanchot ya marca, en 1953, un punto de partida fecundo.5 A partir de allí, es fácil para Blanchot mostrar que para Borges las palabras "truco" o "falsificación" implican algo muy distinto de lo que generalmente pensamos. En vez de negar la dignidad de la literatura, la afirman, porque la dignidad de la literatura no está en la existencia de un gran autor sino en la existencia de una gran literatura.

5 Sobre el tema véase mi libro, El juicio de los parricidas, La nueva generación argentina y sus maestros [Martínez Estrada, Mallea, Borges] (Buenos Aires: Deucalión, 1956). 
Borges comprend que la périleuse dignité de la littérature n'est pas de nous faire supposer au monde un grand auteur, absorbé dans de rêveuses mystifications, mais de nous faire éprouver l'approche d'une étrange puissance, neutre et impersonnelle. (...) l'essentiel, c'est la littérature, non les individus, et dans la littérature, qu'elle soit impersonnellement, en chaque livre, l'unité inépuisable d'un seul livre et la répétition lassée de tous les livres. (118)

La consecuencia narrativa de esta concepción (que Borges mismo ha desarrollado en "La flor de Coleridge", ensayo de Otras inquisicio$n e s, 1952)$, aparece indicada por Blanchot al referirse al extraño relato, "Pierre Menard, autor del Quijote" (está en Ficciones, 1944), en que basta atribuir a un escritor contemporáneo algunos fragmentos de la novela de Cervantes, para que dichos textos cambien de significado. Toda escritura es traducción, apunta Blanchot. Aqui está el germen de un análisis que retomará casi diez años más tarde la crítica francesa y que examinaremos al repasar los trabajos de Gérard Genette. El artículo de Blanchot concluye señalando:

Dans une traduction, nous avons la même oeuvre en un double langage; dans la fiction de Borges, nous avons deux oeuvres dans l'identité du même langage et, dans cette identité qui n'est pas une, le fascinant mirage de la duplicité des possibles. Or, là où il y a un double parfait, l'original est effacé, et même l'origine. Ainsi, le monde, s'il pouvait être exactement traduit et redoublé en un livre, perdrait tout commencement et toute fin et deviendrait ce volume sphérique, fini et sans limites, que tous les hommes écrivent et où ils sont écrits: ce ne serait plus le monde, ce serait, ce sera le monde perverti dans la somme infinie des ses possibles. (Cette perversion est peut-être le prodigieux, l'abominable Aleph.) (118-119)

La paradoja última del análisis de Blanchot es que la literatura no es un simple engaño sino "el peligroso poder de ir hacia lo que es, por la infinita multiplicidad de lo imaginario". En lo imaginario reside lo infinito. Hay en el mismo libro de Blanchot otras referencias a Borges $y$, en particular, a uno de sus textos críticos más importantes, el prólogo a La invención de Morel, de Adolfo Bioy Casares. Publicado en 1940, al frente de esa novela fantástica, el prólogo es casi desconocido para muchos lectores, y aún críticos, de Borges. Es, sin embargo, 
imprescindible para comprender su estética de la narración. Blanchot (como en otro contexto, Robbe-Grillet) le dedica dos veces su atención. En el capítulo titulado muy borgianamente, "Le secret du Golem", Blanchot examina la novela de Bioy y menciona la opinión de Borges sobre ella; en otro capítulo, "Le tour d'écrou", sobre Henry James y sus Notebooks, Blanchot parafrasea algunos conceptos del prólogo a $L a$ invención de Morel, especialmente los que se refieren a la superioridad de la novela moderna en el campo de la invención de tramas y argumentos, lo que los ingleses llaman "the plot". Aquí Blanchot cita a Borges para disentir de él. El ejemplo de Kafka y el ejemplo de James (especificamente: Der Prozes, y The Turn of the Screw), que el propio Borges cita en su prólogo, le sirven para refutar al autor argentino.

No es posible analizar aquí en detalle los argumentos de Blanchot ni presentar los contra-argumentos que un lector de Borges podría sugerir. Baste indicar que la discrepancia central se basa, tal vez, en el hecho de que Blanchot está usando versiones francesas tanto del texto de los Notebooks como del prólogo a La invención de Morel. Una lectura trilingüe de los textos originales permitiria demostrar, creo, que cuando James habla de "subjects", o Borges de "tramas" o "argumentos", la traducción francesa de "fables", o de "sujets", no es siempre iluminadora. Es posible que Blanchot haya sido víctima ' de un ligero error de traducción. Pero tratándose de Borges, qué inevitable parece ser esto. Toda escritura es traducción.

\section{Hacia la lectura como Escritura}

Corresponde a Gérard Genette, en un artículo de L'Herne (1964), llevar a su conclusión crítica una de las observaciones más interesantes de Blanchot: la que se refiere a "Pierre Menard, autor del Quijote". En su primera versión, el artículo de Genette se titula, programáticamente, "La littérature selon Borges". Al recogerlo dos años después en el primer volumen de Figures (1966), el título ha cambiado, tal vez por influencia de Foucault. Ahora se llama, "L'utopie littéraire". (En la versión de 1964 se hablaba a veces de "mythe" donde ahora se habla de "utopie".) Examinaré la versión más reciente porque es la que contiene un desarrollo más completo del punto de vista del crítico. ${ }^{6}$

( Gérard Genette, Figures (Paris: Editions du Seuil, 1966), pp. 123-132. En Critique (núm. 234, Paris, noviembre 1966, pp. 932-939) se publicó una 
Genette empieza por subrayar un aspecto de la obra de Borges que suele desconcertar a ciertos lectores e irritar a no pocos críticos: algunos de sus ensayos, observa, se reducen a un breve catálogo de las diversas entonaciones que han tomado en el curso de los siglos, una idea, un tema, una metáfora. El crítico francés señala por esto que la obra de Borges "semble possédée d'un étrange démon du rapprochement". Buena parte de esta obra crítica aparece dedicada a buscar las fuentes de un texto, o un autor; otra parte a seguir la pista de los precursores. Por esta característica doble, la obra de Borges se hace en parte susceptible de 12 acusación de pedantería. La expresión es de Ibarra en su prefacio a la traducción francesa de Ficciones; Genette la cita para indicar un enfoque posible de este tema: Pero no la acepta.

Mais le goût de rencontres et des parallélismes répond chez Borges à une idée plus profonde, et dont les conséquences nous importent. Cette idée, nous en trouvons une formulation agressive dans le conte "Tlön, Uqubar, Orbis Tertius": "On a établi que toutes les oeuvres sont l'oeuvre d'un seul auteur, qui est intemporel et anonyme." (124)

A partir de este texto, Genette señala en otros de Borges (tomados ya de sus cuentos, ya de sus ensayos) la idea de una literatura como "un espace homogène et réversible où les particularités individuelles et les préséances cronologiques n'ont pas cours". Genette razona que semejante idea puede aparecer a los espíritus positivos como una simple fantasía, o como un puro deseo. Pero señala que para Borges es más bien un mito, "au sens fort du terme, un voeu profonde de la pensée". Después de repasar las dos perspectivas que of rece Borges para explicar esta idea (la panteísta, que cree en la unidad de un Espíritu creador; la clásica, que desdeña discutir la pluralidad de autores), Genette elige una tercera, que le parece más profunda:

Mais l'idée excessive de la littérature, où Borges se complait parfois à nous entrainer, désigne peut-être une tendance profonde de l'écrit, qui est d'attirer fictivement dans sa sphère l'integralité des

interesante reseña del libro de Genette por Henri Ronse en que se vincula la obra crítica de Borges con la del crítico francés. Hay un error lamentable en el primer párrafo de la reseña: Ronse atribuye a Borges una cita de Unamuno que Genette hace en la página 127. Por su parte, y en la misma página, Genette se equivoca dos veces al mencionar el supuesto autor árabe del Quijote: lo llama Cid Hamet Bengeli, con omisión de algunas letras. 
choses existantes (et inexistantes), comme si la littérature ne pouvait se maintenir et se justifier à ses propres yeux que dans cette utopie totalitaire. Le monde existe, disait Mallarmé, pour aboutir à un Livre. (126)

Hasta aqui Genette no ha hecho sino glosar muchos enfoques que Borges ofrece en algunos cuentos y sobre todo en dos ensayos de Otras inquisiciones ("La flor de Coleridge", "Del culto de los libros") que también había utilizado parcialmente Blanchot. Pero a partir de aquí, Genette efectuará una lectura más acuciosa de "Pierre Menard, autor del Quijote", lo que le permitirá avanzar un poco más sobre lo observado por Blanchot. En aquel cuento encontrará Genette la base para afirmar que la lectura es

...l'opération la plus délicate et la plus importante de toutes celles qui contribuent à la naissance d'un livre:... (129)

Al glosar el cuento, así como los textos mencionados de Olras inquisiciones, Genette subraya la coincidencia del punto de vista de Borges con el de Valéry (lo que también habia hecho al pasar Blanchot). Pero agrega elementos tomados de un tercer artículo del citado libro de ensayos: "Kafka y sus precursores", en que Borges llega a la conclusión de que "cada escritor crea a sus precursores. Su labor modifica nuestra concepción del pasado, como ha de modificar el futuro." Al citar este fragmento de Borges en su estudio, omite Genette indicar que en el texto hay una llamada que remite al lector a un libro de T. S. Eliot, Points of View (1941). Al no reparar en la cita, Genette se priva de comprobar que el germen del punto de vista de Borges ya estaba en un famoso ensayo de Eliot, "Tradition and the individual talent", ensayo verdaderamente germinal para esta teoría de la impersonalidad de la tradición literaria, y de su reversibilidad. Más inexcusablemente que Genette (al fin y al cabo, especialista en literatura francesa), el crítico inglés Harold Bloom también cita en su excelente libro sobre Yeats, aquel texto de Borges y también pasa por alto la referencia a Eliot. ${ }^{7}$

Volviendo a Genette, hay que subrayar que este enfoque de Borges le permite esbozar la "admirable utopía" que encierra: mito, agrega, que contiene más verdad que las verdades de nuestra "ciencia" literaria.

7 Harold Bloom, Yeats (New York: Oxford University Press, 1970), p. 4. La omisión de Bloom podría explicarse por la escasa simpatía que le despier. ta Eliot. 
La littérature est bien ce champ plastique, cet espace courbe où les rapports le plus inattendus et les rencontres les plus paradoxales sont à chaque instant possibles. (...) La genèse d'une oeuvre, dans le temps de l'histoire et dans la vie d'nn auteur, est le moment le plus contingent et le plus insignifiant de sa durée. - (...) Le temps des oeuvres n'est pas le temps défini de l'écriture, mais le temps indéfini de la lecture et de la mémoire. Le sens des livres est devant eux et non derrière, il est en nous: un livre n'est pas un sens tout fait, une révélation que nous avons à subir, c'est une réserve de formes qui attendent leur sens, c'est "l'imminence d'une révelation qui ne se produit pas", et que chacun doit produire pour lui-même. (131-132)

Las palabras de Borges que Genette cita en este último párrafo pertenecen al primer ensayo de Otras inquisiciones ("La muralla. \& los libros") y sirven para concluirlo. Luego continúa el crítico francés:

Tous les auteurs sont un seul auteur parce que tous les livres sont un seul livre, d'où suit encore qu'un seul livre est tous les livres, "et j'en sais qui, à l'égal de la musique, sont tout pour tous les hommes." La bibliothèque de Babel est parfaite ab aeterno; c'est l'homme, dit Borges, qui est un bibliothécaire imparfait; parfois, faute de trouver le livre qu'il cherche, il en écrit un autre: le même, ou presque. La littérature est cette tâche imperceptible -et . infinie. (132)

Por el camino de la identificación entre todos los libros y todos los autores, Genette ha retornado a la noción de infinito de la que había. partido Blanchot. La novedad de su enfoque, con respecto al de éste, es insistir más en el análisis puramente literario. Además, en los últimos párrafos de su ensayo se encuentra implícita la concepción de la escritura como lectura que aparece como tema central en la especulación crítica de Borges. En otro lugar he analizado detenidamente este tema. ${ }^{8}$

\section{Fuera de la Circularidad}

En el mismo volumen de L'Herne en que apareció la primera versión del texto de Genette, se publicó uno muy breve de Jean Ricardou, "The

8 Véase mi ensayo "Borges: The Reader as Writer", que se publicará próximamente en un número dedicado a Borges que prepara Tri-Quarterly bajo la dirección de Mary Kinzie. 
God of the Labyrinth", que es como la semilla de muchas páginas de su futuro libro, Problèmes du nouveau roman (1967)..$^{9}$ Ricardou parte de la observación de que para algunos el carácter laberíntico de las ficciones de Borges no es sino la ocasión de un juego amable, a veces inquietante, pero que no pone en cuestión el sentido común ni la naturaleza del mundo cotidiano. Para otros, en cambio, "le labyrinthe et le monde du bon sens se mettent en cause réciproquement (symétriquement)". De ahí päa Ricardou a enunciar brevemente algunas características de los relatos borgianos.

Le récit borgésien dont l'apparence est rassurante, univoque, se trouve insidieusement gauchi par de menues anomalies capables d'en assurer la duplicité. Souvent (exceptons l'ambigüité foudroyante du "Sorcier ajourné"), un commentaire paraît requis pour les révéler: celui qui dégage les imperceptibles irrégularités du récit de Cartaphilus ("l'Immortel"), celüi qui soulignerait les variantes caractéristiques des deux versions (françaises) de l'Appro. che du Caché. (125)

Antes de seguir adelante, conviene señalar que uno de los textos que Ricardou atribuye a Borges ("El brujo postergado") pertenece al Libro de Petronio, del Infante Juan Manuel, como lo indica el propio Borges en Historia Universal de la Infamia. En el prólogo a la primera edición de este libro (1935), Borges apunta que no tiene otro derecho sobre éste $\mathrm{y}$ otros textos semejantes, "que los de traductor y lector". ${ }^{10}$ éste y otros textos semejantes, "que los de traductor y lector": $:^{10} \mathrm{Su}$ "traducción" del ilustre relato del siglo xIV ha consistido principalmente en modernizar la ortografía y podar un poco el texto. Es claro que Ricardou podría alegar, en la mejor tradición borgiana, que como lector y traductor, Borges es autor del relato.

Pero volvamos a la nota de Ricardou. El crítico francés termina observando que al abandonar la idea de una solución privilegiada, al postular Borges en sus cuentos la naturaleza profundamente laberíntica de toda ficción,

le transit d'une solution à telle ou telle autre $(\ldots)$ s'affirme non plus comme dévoiement frappé d'hésitations, mais, liberé, comme un moyen de recherches, d'inédites mises en contact, de création.

9 Jean Ricardou: "The God of the Labyrinth", en L'Herne (Paris, 1964), pp. $125-126$.

10 Jorge Luis Borges, Historia universal de la infamia (Buenos Aires: Editorial Tor, 1935), p. 6. 
Ricardou concluirá su breve nota vinculando la obra de Borges a la de los escritores del Nouveau Roman: Robbe-Grillet, Butor, Simon:

...Borges se trouve au centre de leurs préoccupations. Ils se veulent inspirés par The God of the Labyrinth. (126)

En un artículo de 1967, que publica la revista Critique, y que será incorporado más tarde a Problèmes du nouveau roman, como epílogo, vuelve Ricardou a examinar unos textos de Borges. El artículo se titula, "Le caractère singulier de cette eau", y se basa principalmente en un texto de The Adventures of Artbur Gordon Pym, sobre el que Borges (entre otros) había llamado la atención. ${ }^{11}$ El texto de Poe se concentra principalmente en un episodio: navegando hacia el Polo Sur, los tripulantes de la goleta Jane-Guy llegan a una tierra extraña y allí encuentran una agua, más extraña aún cuyo aspecto parece corrompido. Luego de haber resumido el episodio, y citado in extenso el pasaje pertinente, examina Ricardou la opinión de Marie Bonaparte (en su famoso estudio psicoanalítico sobre Poe) y la de Gaston Bachelard, en L'Eatu et les Rêves. En ambos críticos Ricardou cree reconocer dos características comunes: (a) el escamoteo de una parte del texto que no encaja en su teoría; (b) la idea de que la literatura tiene por fin "exprimer un antécedenl". Así, para Marie Bonaparte, esa agua es sangre; para Bachelard, leche; para ella, el texto expresa el inconsciente de los hombres; para él, los sueños que sirven de prefacio a las obras. Ricardou no acepta estas explicaciones parciales, o dirigidas a algo fuera de la literatura.

Mais on peut douter que la litérature soit, fût-elle relative, cette transparence qui livre autre chose. Il est possible que le texte présente au contraire une fondamentale opacité, et soit l'endroit du permanent problème. Au lieu de fluir incessament la page au profit d'un quelconque antécédent fixe, l'exégese serait alors prise dans une inlassable circularité. (198)

Ya en su breve texto sobre Borges para L'Herne, Ricardou habia subrayado esta circularidad de la literatura borgiana:

11 Jean Ricardou: Problèmes du nouveau roman (Paris: Editions du Seuil, 1967), pp. 193-207. Ricardou cita el título de la novela de Poe, según la versión francesa: Les Aventures d'Artbur Gordon Pym. 
Les différentes voies de leurs labyrinthes sont essentiellement les diverses plans de réalité (monde courant, souvenirs, phantasmes, rêves...) entre certains desquels s'accomplit une circulation nouvelle, intense, qui confine parfois à la contamination réciproque. (126)

Es la misma circularidad de la literatura borgiana la que ahora Ricardou busca determinar en el enigma planteado por el texto de Poe. Para resolverlo habrá de acudir precisamente a un comentario de Borges sobre dicho texto. Está en el ensayo, "El arte natrativo y la magia", recogido en el volumen, Discusión (1932).$^{12}$ Para Borges es indudable que

El secreto argumento de esa novela es el temor y la vilificación de lo blanco. Poe finge unas tribus que habitan en la vecindad del Círculo Antártico, junto a la patria inagotable de ese color, y que de generaciones atrás han padecido la terrible visitación de los hombres y de las tempestades de la blancura. El blanco es anatema para esas tribus y puedo confesar que lo es también, cerca del último renglón, del último capitulo, para los condignos lectores. Los argumentos de ese libro son dos: uno inmediato, de vicisitudes marítimas; otro infalible, sigiloso y creciente, que sólo se revela al final. (114-115)

Aquí practica Borges una digresión, en que aprovecha para mencionar una opinión de Mallarmé y unos versos suyos, un capítulo de Melville sobre la blancura de Moby Dick, para volver a la elucidación de su segundo argumento.

Imposible exhibir o analizar aquí la novela entera; básteme traducir un rasgo ejemplar, subordinado - como todos- al secreto argumento. Se trata de la oscura tribu que mencioné y de los riachuelos de su isla. Determinar que su agua era colorada o azul, hubiera sido recusar demasiado toda posibilidad de blancura. Poe resuelve este problema asi, enriqueciéndonos: $(115-116)$

Borges intercala aqui una extensa cita del pasaje que Ricardou estaba precisamente analizando. Es obvio por la cita, y sobre todo por

12 Jorge Luis Borges, Discusión (Buenos Aires: Gleizer Editor, 1932), pp. 109-124. Hay pequeñas variantes en el texto de Borges y en la traducción de Poe entre esta primera edición y la corriente de Emecé, en la colección de sus Obras completas. Pero el estudio de las mișmas escapa a estẹ artículo, 
el lugar de su ensayo en que aparece inserta, que para Borges el aspecto singular de esta agua tiene un propósito: omitir la mención del color blanco. La frase clave, en este sentido, sería: "No era incolora ni era de un invariable color, ya que su fluencia proponía a los ojos todos los matices del púrpura, como los tonos de una seda cambiante." ${ }^{13}$ Los demás atributos de esa agua misteriosa, le interesan menos a Borges. O dicho de otro modo: él quiere determinar una sola cosa: la ausencia de toda mención del blanco.

Al analizar este pasaje de Borges, Ricardou observa con justeza que tres puntos incitan a la controversia. En primer lugar, le parece una tentativa de reducción realista afirmar que el blanco era anatema en esa isla porque los habitantes de ella habrían sufrido la incursión de hombres y tempestades blancas. Ricardou cita un pasaje de Poe que contradice esa interpretación: "Era positivamente evidente que ellos nunca habian visto ningún individuo de raza blanca." ${ }^{14}$ En segundo lugar, Ricardou comenta in extenso otros pasajes del libro en que Poe omite mencionar el blanco, ya sea por cambiar el color habitual de un objeto (albatros negros), ya por escamoteo (los labios negros de los insulares esconden sus dientes), ya por silencio (no se menciona el color de la esclerótica de los habitantes de la isla). Pero la más importante de las confusiones de Borges es la tercera: no advertir que el carácter del agua común no es la blancura sino la transparencia. Sólo cuando es espuma (observa Ricardou) el agua es blanca. A partir de estas observaciones, el crítico construye toda una teoría sobre el texto de Poe, teoría que le permite llegar a la conclusión de que esa agua extraña es una escritura, y que toda la región austral es una página escrita. De ahí las últimas palabras de su epílogo, y del libro:

L'ultime aventure d'Arthur G. Pym, en symbolisant une page d'écriture, c'est-à-dire le lieu et l'acte qui l'instituent, nous assure que par la fiction, la littérature n'emprunte au monde des matériaux que pour se désigner elle-même. C'est telle circularité, et l'étrange vide moyeu autour duquel s'agencent les signes, que ne

13 Cf. Discusion, p. 116. El texto original de Poe dice: "It was not, colorless, nor was it of any one uniform color - presenting to the eye, as it flowed, every possible shade of purple, like the hues of a changeable silk". Cf. Edgar Allan Poe: The Norrative of Artbur Gordon Pym (New York: Hill and Wang, 1966), p. 151.

14 El texto de Poe dice: "It was quite evident that they had never before seen any of the white race -from whose complexion, indeed they appeared to recoil". (Narrative, p. 147). Como se adverticá, Ricardou no çita la última parte de la frase. 
doit jamais perdre de vue toute lecture en altitude: "Le langage se réflechissant". (Mallarmé) (207)

Lo cual está bien, y hasta muy bien, pero tiene poco que ver con lo que Borges buscaba en su ensayo sobre "El arte narrativo y la magia". Es posible que Borges se equivoque en cuanto al significado del agua extraña, y su confusión entre blancura y transparencia es característica en un hombre perseguido por la ceguera. Pero la cita de Poe en su ensayo cumple una función distinta de la que cumple en el ensayo de Ricardou, y esto sólo basta para alterar profundamente su significado. A Ricardou no le interesa el argumento que Borges expone en su ensayo; le interesa en cambio su propio argumento. Al no examinar el de Borges, le atribuye intenciones y errores que no son tales. Para comprender mejor esto conviene repasar el texto completo de Borges.

Su ensayo comienza señalando (en 1932, no hay que olvidar) que el "análisis de los procedimientos de la novela ha conocido escasa publicidad". Para remediar en algo esa carencia, él of recerá algunas verificaciones (la palabra es suya). Comienza por examinar la "faz novelesca" de The Life and Death of Jason, libro que William Morris publica en 1867. Luego examina The Narrative of $A$. Gordon Pym, que Poe publica en 1838. El resultado de este doble análisis es resumido así:

Rectamente se induce de lo anterior que el problema central de la novelística es la causalidad. Una de las variedades del género, la morosa novela de caracteres, finge o dispone una cancatenación de motivos que se proponen no diferii de los del mundo real. Su caso, sin embargo, no es el común. En la novela de continuas vicisitudes, esa motivación es improcedente, y lo mismo en el relato de breves páginas y en la infinita novela espectacular que compone Hollywood con los plateados idola de Joan Crawford y que las ciudades releen. Un orden muy diverso los rige, lúcido $y$ atávico. La primitiva claridad de la magia. (88)

El resto del artículo examina otros aspectos del mismo tema, cita un pasaje de Frazer, examina textos antropológicos, evoca brevemente algunos relatos de Chesterton y algunos films de von Sternberg, para concluir con dos observaciones importantes, que aparecen separadas en el texto pero que por razones de economía reuno aquí:

, . la magia es la coronación o pesadilla de lo causal, no su con. 
tradicción. El milagro no es menos forastero en ese universo que en el de los astrónomos. Todas las leyes lo rigen, y otras imaginarias. Para el supersticioso, hay una necesaria conexión no sólo entre un balazo y un muerto, sino entre un muerto y una maltratada efigie de cera o la rotura profética de un espejo o la sal que se vuelca o trece comensales terribles.

Esa peligrosa armonía, esa frenética y precisa causalidad, manda en la novela también. (...) Ese recelo de que un hecho temible pueda ser atraído por su mención, es impertinente en el asiático desorden del mundo real, no así en una novela, que debe ser un juego preciso de vigilancias, ecos y afinidades. Todo episodio, en un cuidadoso relato, es de proyección ulterior. ( $\ldots$ ) Esa teleología de palabras y de episodios es omnipresente también en los buenos films. (...) Pero la ilustración más cabal de un orbe autónomo de corroboraciones, de presagios, de monumentos, es el predestinado Ulises de Joyce. Basta el examen del libro expositivo de Gilbert o, en su defecto, de la vertiginosa novela.

Procuro resumir lo anterior. He distinguido dos procesos causales: el natural, que es el resultado incesante de incontrolables e infinitas operaciones; el mágico, donde profetizan los pormenores, lúcido y limitado. En la novela, pienso que la única posible họradez está con el segundo. Quede el primero para la simulación psicológica. (89-91)

Leído en el contexto del artículo entero, el análisis a que Borges somete el texto de Poe adquiere un sentido diferente del que Ricardou le atribuye. Es posible que Borges se haya equivocado en cuanto al significado exacto del agua misteriosa. Lo cierto es que no se equivocó en cuanto a la naturaleza del texto de Poe. En esta novela, los pormenores profetizan, como lo demuestra tan convincentemente Ricardou en su precisa lectura; no hay en ella nada dejado al azar: la causalidad la rige. $\mathrm{Y}$ eso es precisamente lo que Borges quería demostrar. Su ensayo, por otra parte, es el antecedente obligado del prólogo a La invención de Morel, y contribuye a explicarlo. Seguramente que si Maurice Blanchot lo hubiese leído habría podido comprender mejor a qué se refería Borges en el prólogo al hablar de "tramas" o "argumentos". No se trata simplemente de un "sujet". Se trata de esa causalidad mágica en que los pormenores profetizan, en que todo episodio es de proyección ulterior, en que la narración se convierte en un juego preciso de vigilancias, ecos y afinidades. Ya en 1932, a punto de comenzar su carrera 
de narrador mágico, Borges estaba fijando las coordenadas retóricas que sus ficciones habrían de ilustrar.

El desconocimiento de este texto por parte de Blanchot es explicable. Su libro se publicó en 1959; la primera versión francesa del ensayo de Borges, hecha por Philippe Sollers, se publicó en el número 7 de Tel Quel (Otoño 1961). Lo que es menos excusable es la desatención prestada por Ricardou al resto del ensayo, ya que él está trabajando con el texto completo, tal como apareció en la versión francesa de Discusión. Más excusable aún es la omisión de Ricardou si se tiene en cuenta que ella está en conflicto con su propia teoría de la circularidad de la literatura. No advierte que el texto crítico de Borges sobre Poe se remite sobre todo a sí mismo.

\section{El Complot Omitido}

En el número de l'Heme, tantas veces citado, se encuentra asimismo un trabajo de Clande Ollier, titulado "Theme du texte et du complot", que se concentra sobre todo en un análisis del relato, "Tema del traidor y dèl héroe", de Ficciones. ${ }^{15} \mathrm{La}$ observación inicial más importante de Ollier se refiere a la existencia de dos conspiraciones en dicho relato: una, a nivel del argumento, en que Fergus Kilpatrick es el "héroe"; la otra es la que está al nivel de la "escritura"; en que el narrador se llama Ryan:

En fait, ce n'est pas une, mais deux conspitations qui vont s'ourdir de concert, simultanément inscrites sous les mêmes mots: celle quotidienne dont Fergus Kilpatrick fut le "glorieux capitaine", celle scripturale dont Borges, presque entièrement dissimulé derrière Ryan, se fait le probe et minutieux révélateur. (277)

Después de analizar al detalle las sucesivas versiones del complot, Ollier llega a la conclusión:

Ici, cinq drames sont superposés, dont le texte rebrousse la chronologie: la rédaction de Borges, l'investigation de Ryan, l'improvisation de Nolan, l'élaboration de Shakespeare, l'assassinat de

\footnotetext{
15 Claude Ollier, "Thème du texte et du complot", en L'Herne, pp. 276 279. Sobre la adantación cinematográfica de este cuento por Bernardo Berto. lucci, véase: Richard Roud: "Fathers and Sons", en Sight and Sound (vol. 40, No 2, London. Sping 1971), pp. 61-64.
} 
Jules César. Et cins scènes, l'une aprés l'autre devoilées et occupèes: une page blanche, des archives truquées, une grande ville moderne, un théâtre classique, la Rome antique. (...)

$\mathrm{Si}$ ce texte est pour nous si important, pardelà la concision et l'ironie de son éclat que Borges y monte et démonte sous nos yeux les rouages de la machine, montre comment les mots l'alimentent et assurent sa bonne marche, sécretant une bistoire et sécretant l'Histoire. "Thème du traître et du héros" est un abrége du mécanisme - constitutif et fonctionnel- de toute fiction. (278)

En el resto del artículo, Ollier examina las posibilidades teatrales y cinematográficas del cuento que habría de ser filmado en 1970 por Bernardo Bertolucci, bajo el título de La stronegia del ragno. También señala Ollier la existencia de un film francés, Paris nous appartient, de Jacques Rivette (1958), en que hay un tema vecino al del cuento de Borges. Uno de los personajes del film, Anne, tiene entre sus libros de cabecera un ejemplar de la traducción francesa de Otras inquisiciones, junto a un ejemplar de Shakespeare.

El artículo de Ollier aporta interesantes perspectivas a la lectura del "Tema del traidor y del héroe". Córrobora, además, el predicamento que tiene Borges entre los practicantes del Nouveau Roman. Pero es, a su vez, víctima de un error de interpretación. No advierte que el cuento de Borges no sólo indica las semejanzas entre el destino de Kilpatrick y el de Julio César, y entre los textos de Nolan y de Shakespeare, pero también entre el destino de Kilpatrick y el (futuro) de Lincoln, así como alude, en su entrelínea a otro famoso Héroe y otro famoso rela. to: Jesús en los Evangelios. Para establecer el vínculo secreto entre la interpretación del "Tema del traidor y del héroe" con la historia evangélica sólo hay que releer cuidadosamente el penúltimo párrafo del cuento a la luz de lo que dice Borges en otro relato, "Tres versiones de Judas" que se encuentra en el mismo volumen de Ficciones, apenas separado de aquél por otros dos cuentos. En la última de las tres versiones de Judas, el verdadero Redentor es el traidor. Ollier pudo haber enriquecido su lectura del "Tema del traidor y del héroe" si hubiera seguido excavando los otros niveles de significación.

\section{Un Discípulo de Althusser}

En un artículo publicado en Les Temps Modernes (1966) y recogido luego en un libro del mismo año, pout whe théprie de la production lit- 
térare, ${ }^{16}$ Pierre Macherey examina el tema de "Borgès et le récit fictif". Macherey pertenece al grupo de Louis Althusser, que se ha especializado en una nueva lectura de Karl Marx, a la luz de una concepción estructural de la sociedad y de la historia. Lo que se propone Macherey en su libro es (nada menos) que examinar las condiciones de la producción literaria, la especificidad del discurso literario en relación con el discurso ideológico, el mecanismo de la complejidad literaria. $\mathrm{O}$, como han indicado François Wahl en una reseña extremadamente polémica de este libro que ha publicado Critique, ${ }^{17}$ el propósito de Macherey es soumettre à une élaboration scientifique la pratique qui transforme un discours en littérature. (537)

Macherey parte de la obvia comprobación de que Borges se plantea los problemas del relato de una manera profundamente ficticia, que lo que él nos propone es una teoría ficticia del relato. Siguiendo a Blanchot, también señala el juego de las paradoxas del infinito, y la idea obsesionante de un libro, a la vez necesario y múltiple. También reconoce la importancia de la operación de leer, la existencia dentro de cada relato de distintas versiones del mismo, etc., etc. La conclusión de esta primera etapa del análisis es:

Le problème semble ainsi clairement posé: ou bien il y a un sens du récit, et la fausse résolution est une allégorie, bien il n'y en a pas, et la fausse résolution est une allégorie de l'absurdité. C'est bien ainsi qu'on interprète généralement l'oeuvre de Borgès: on l'achève en lui attribuant le tournures d'un scepticisme intelligent. Il n'est pas certain que le scepticisme soit intelligent, ni que le sens profond des récits de Borgès soit dans leur raffinement apparent. (280)

Esta conclusión le parece falsa a Macherey. Según él, habría que buscar el sentido del texto borgiano no en la lectura sino en la escritura. Borges usa la alusión para indicar un texto más que para redactarlo; en vez de trazar la línea del relato, prefiere marcar la posibilidad de ese relato, posibilidad siempre postergada, remisa. Lo mismo pasa con sus artículos de crítica que son también ficticios, aunque se ocupen de obras y autores reales. La conclusión, según Macherey:

1) Pierre Macherey: Pour une théorie de la production littéraire (Paris: François Maspéro, 1966), pp. 277-285.

17 François Wahl, "Littérature, science, idénlogie", en Critiqüe (núm. 241, Paris, Juin 1967), pp. 536-543, 
...c'est pourquoi ses articles de critique, même lorsqu'ils portent sur des oeuvres réelles, sont fictifs; c'est pourquoi aussi ses récits de fiction ne valent que par la critique explicite qu'ils contien. nent d'eux-mêmes. (281)

El análisis somero de algunos relatos representativos de Ficciones ("La forma de la espada", "El jardín de senderos que se bifurcan", "La biblioteca de Babel", "Tlön, Uqbar, Orbis Tertius") permiten a Macherey concluir que el mito del laberinto

correspond à l'idée d'un récit completement objectif, qui prendrait à la fois tous les partis, et les développerait jusqu'à leur terme; mais ce terme est impossible, et le récit ne donne jamais que l'image du labyrinthe, parce que, condammné a choisir un terme défini, il est obligé de dissimuler toutes les bifurcations, et de les noyer dans la ligne d'un discours. (...) Chaque récit particulier trahit l'idée du labyrinthe, mais il nous en donne le seul reflet lisible. (...) La vrai labyrinthe c'est qu'il n'y a plus de labyrinthe: écrire, c'est perdre le labyrinthe. (...) Cette entreprise peut être tenue à la fois pour une réussite et pour un échec, dans la mesure où à travers les insuffisances d'un récit, Borgès parvient à nous montrer que nous n'avons rien perdu. (284-285)

Aunque el esfuerzo de reducción de la obra de Borges a un modelo coherente que realiza Macherey es interesante, tiene el inconveniente de ser demasiado simplista. En un nivel superficial, reduce la complejidad y variedad de los relatos borgianos a un modelo único. Si lo que dice Macherey puede aplicarse a los relatos que él cita, no parece posible extenderlo a toda su obra (ficción, crítica, pocsía). Por otra parte, la importancia de la noción de lectura en Borges, que ha sido subrayada entre otros por Gérard Genette, se contradice explícitamente con algunos postulados del modelo de Macherey. Finalmente, su punto de partida es discutible. Como ha señalado François Wahl:

En choisissant les structures de l'idéologie contre celles de l'écriture, Macherey ne décale pas, il rédnit: exactement comme qui prétendrait fonder la science des rêves non dans l'organisation de l'inconscient mais dans ce qui s'y représente du corps. (542) 


\section{LA Heterotopia Borgiana}

He dejado deliberadamente para el final el texto que me parece más interesante de todos: el Préface a Les Mots et les Choses, de Michel Foucault. ${ }^{18} \mathrm{Al}$ presentar su libro, Foucault empieza por reconocer su deuda con el narrador argentino:

Ce livre a son lieu de naissance dans un texte de Borges. Dans le rire qui secoue à sa lecture toutes les familiarités de la pensée - de la notre: de celle qui a notre ãge et notre géographie-, ébranlant toutes les surfaces ordonnées et tous les plans qui assagissent pour nous les foisonnement des êtres, faisant vaciller et inquiétant pour longtemps notre pratique millénaire du Même et de l'Autre. Ce texte cite "une certaine encyclopédie chinoise" où il est écrit que "les animaux se divisent en: a) appartenant à l'Empereur, b) embaumés, c) apprivoisés, d) cochons de lait, e) sirènes, f) fabuleux, $\mathrm{g}$ ) chiens en liberté, h) inclus dans la présente classification, i) qui s'agitent comme des fous, j) innombrables, $\mathrm{k}$ ) dessinés avec un pinceau très fin en poils de chameau, 1) et caetera, m) qui viennent de casser la cruche, n) qui de loin semblent des mouches". Dans l'émerveillement de cette, taxino. mie, ce qu'on rejoint d'un bond, ce qui, à la faveur de l'apologue, nous est indiqué comme le charme exotique d'une autre pensée, c'est la limite de la nôtre: l'impossibilité nue de penser cela. (7)

Al meditar sobre este texto de Borges, Foucault descubre que lo que le choca no es "la bizarrerie des rencontres insolites", sino otra cosa:

La monstruosité que Borges fait circuler dans son énumeration consiste au contraire en ceci que l'espace commun des rencontres s'y trouve lui-même ruiné. (...) Borges n'ajoute aucune figure à l'atlas de l'impossible; il ne fait jaillir nulle part l'éclair de la rencontre poétique; ils esquive seụlement la plus discrète mais la plus insistente des nécessités; ils soustrait l'emplacement, le sol muet où les êtres peuvent se juxtaposer. (...) Ce texte de Borges m'a fait rire longtemps, non sans une malaise certain et difficile à vaincre. Peut.être parce que dans son sillage naissait le soupçon qu'il y a pire désordre que celui de l'incongru et du rapproche-

18 Michel Foucault: Les mots et les choses (Paris: Gallimard, 1966), pp. 7-16. 
ment de ce qui ne convient pas; ce serait le désordre qui fait scintiller les fragments d'un grand nombre d'ordres possibles dans la dimension, sans loi ni géométrie, de l'bétéroclite; et il faut entendre ce mot au plus prés de son etymologie: les choses y sont "couchées", "posées", "disposées" dans des sites à ce point différents qu'il est impossible de trouver pour eux un espace d'accueil, de définir au-dessous des uns et des autres un lieu commun. (8-9)

Aquí se encuentra, pues, el punto de partida para Foucault, y el resultado es el libro que sigue. Pero para el lector de Borges, el análisis de Foucault es también un punto de partida, aunque hacia un diferente destino. Hay que observar, en primer término, que tal vez Foucault debió haber señalado, con mayor precisión, que el texto qué él atribuye a Borges es atribuido por Borges ("El idioma analítico de John Wilkins", en Otras Inquisiciones) al Dr. Franz Kuhn, que a su vez lo atribuye "a cierta enciclopedia china que se titula Emporio celestial de conocimientos benévolos".19 Encontramos aquí el recurso, típicamente borgiano, de la mise en abîme: la perspectiva infinita de textos que remiten a textos que remiten a textos. También encontramos en la actitud de Foucault de negarse a asumir esa perspectiva algo vertiginosa, un eco de la reacción de Genette (al no reparar que Borges estaba citando a Eliot) o de Ricardou (al no reconocer el origen del relata, "El brujo posterga. do"). Pero esta observación es lateral. Si la hago es śólo para mostrar una vez más que la naturaleza verdaderamente laberíntica de los textos borgianos es difícil de reconocer aún para aquellos más dispuestos a aceptarla.

La segunda observación se refiere a algo que Foucault indica en el primer párrafo de su Préface y que se convertirá en uno de los centros de su libro: nuestra práctica milenaria del Mismo y del Otro. Es probable que al redactar su Préface, Foucault no supiera que dos años antes Borges había titulado la última sección de su Obra poética (1964), con esas dos palabras, pero en este orden: "El Otro, el Mismo". 20 Sea como fuere, Foucault pone aquí al descubierto uno de los temas centrales, si no

19 Jorge Luis Borges: Otras inquisiciones (Buenos Aires: Editoral Sur, 1952), pp. 123-124.

${ }_{20}$ Jorge Luis Borges, Obra poética 1923-1964 (Bùenos Aires: Emecé Editores, 1964), p. 133. 
el tema central, de la obra de Borges. No es extraño que su Préface contenga por eso mismo una de las caracterizaciones generales más penetrantes de esa obra:

Les utopies consolent: c'est que si elles n'ont pas de lieu réel, elles s'épanouissent pourtant dans un espace merveilleux et lisse; elles ouvrent des cités aux vastes avenues, des jardins bien plantés, des pays faciles, même si leur accès est chimérique. Les bétérotopies inquietent, sans doute parce qu'elles empêchent de nommer ceci et cela, parce qu'elles brisent les noms communs ou les enchevêtrent, parce qu'elles ruinent d'avance la "syntaxe", et pas seulement celle qui construit les phrases, - - celle moins manifeste qui fait "tenir ensemble" (à côté et en face les uns des autres) les mots et les choses. C'est pourquoi les utopies permettent les fables et les discours: elles sont dans le droit fil du langage, dans la di. mension fondamentale de la fabula; les hétérotopies (comme on en trouve si fréquemment chez Borges) dessèchent le propos, arrêtent les mots sur eux-mêmes, contestent dès sa racine, toute possibilité de grammaire; elles dénouent les mythes et frappent de sterilité le lyrisme des phrases. (9-10)

Qué lejos se está en este análisis del esfuerzo tranquilizador de un Macherey que reduce Borges a un modelo, ideológicamente neutro, ineficaz. Para Foucault, Borges pone todo en cuestión al poner en cuestión la sintaxis, la gramática, el lenguaje. Al vincular la contestación de Borges con la que ocurre en ciertos casos de afasia, Foucault llega a decir:

La gêne qui fait tire quand on lit Borges est apparentée sans doute au profond malaise de ceux dont le langage est ruiné; avoir perdu le "commun" du lieu et du nom, Atopie, aphasie. (10)

En esta lectura de Foucault existe ya una posibilidad de acceso a Borges que las otras lecturas francesas no hacían sino buscar. En cietto sentido, Foucault va incluso más lejos que Blanchot y Genette, ya que éstos sólo intentaron revelar algunos de los conceptos subyacentes en la obra de Borges. Foucault, en cambio, apunta al centto de la escritura borgiana: una empresa literaria que se basa en la "total" destrucción 
de la literatura y que a su vez (paradójicamente) instaura una nueva literatura; una "écriture" que se vuelve sobre sí misma para recrear, de sus propias cenizas, una nueva manera de escribir; un fénix, ay, no demasiado frecuente. ${ }^{21}$

EMir Rodríguez MONEGAL

Yale University.

21 Una versión en inglés de este trabajo, hecha por Roberto González Eche. verria, se publicó en la revista Diacritics (vol. 2, number 2, Winter 1972, Ithaca, N. Y.), pp. 27-34. 